\title{
Le rôle des légendes dans le culte de Ghāzī Miyāñ
} (Inde)

The Role of Legend in the Cult of Ghāzì Miyāãn (India)

Delphine Ortis

\section{OpenEdition}

12 Journals

Édition électronique

URL : https://journals.openedition.org/clo/2685

DOI : $10.4000 /$ clo.2685

ISSN : 2266-1816

Éditeur

INALCO

\section{Édition imprimée}

Date de publication : 1 janvier 2016

ISBN : 9782858312337

ISSN : 0396-891X

\section{Référence électronique}

Delphine Ortis, "Le rôle des légendes dans le culte de Ghāzī Miyāñ (Inde) », Cahiers de littérature orale [En ligne], 79 | 2016, mis en ligne le 20 décembre 2016, consulté le 01 juillet 2021. URL : http:// journals.openedition.org/clo/2685; DOI : https://doi.org/10.4000/clo.2685

\section{(ब) $(1) \Theta$}

Cahiers de littérature orale est mis à disposition selon les termes de la Licence Creative Commons Attribution - Pas d'Utilisation Commerciale 4.0 International. 


\title{
Le rôle des légendes dans le culte de Ghāzī Miyāñ (Inde)
}

\author{
Delphine OrTIS
}

Si l'on écoute Oscar Calavia Sáez (1996), « Les saints existent d'abord par le récit ». Les plus populaires concernant le martyr Ghāzī Miyāñ (ou Syed Sālār Mas'ūd Ghāzī), ici sujet de réflexions, parlent de ses trois premiers miracles récompensés chaque fois par l'embellissement de son tombeau et l'accroissement de la popularité de son culte, lui-même résultat des dits miracles. Dans la société indo-musulmane, où il n'existe pas de corps religieux institué reconnu par tous, qui donnerait le la sur qui est saint ou ne l'est pas, leur reconnaissance passe par la conviction dans leur capacité à accomplir des miracles, dont la production est favorisée par l'accomplissement d'un ensemble de rites spécifiques à chacun d'eux. Les saints existent ici d'abord par l'institution d'un culte (Jamous, 1995, p. 43) que diffusent, à qui veut les entendre, les légendes.

Si celles-ci participent bien de la fabrique de la sainteté, nous leur accordons néanmoins un rôle moindre que la littérature sur le sujet, car en discutant avec les adeptes du culte aux saints on se rend rapidement compte que la grande majorité d'entre eux a une connaissance fragmentaire, partielle, voire nulle de la vie des saints qu'ils cherchent à se rendre propice par le culte. Le saint est avant tout pour eux un mort puissant ayant la capacité de modifier le cours des évènements ${ }^{1}$. Il importe donc aux dévots de suivre correctement les rites qui obligent le saint à exaucer leurs vœux ; pour cela, nul besoin de connaître les détails de sa destinée.

1. Les nombreux exemples développés dans le livre de H. Chambert-Loir et C. Guillot (1995) montrent que le saint musulman est plus un être puissant qu'un être parfait (comme dans le christianisme, voir introduction CentLIVRes, 2001) ; voir aussi D. ORTIS (2016 c). 
Le saint peut être aussi prétexte à l'adoration d'un site remarquable, comme l'a montré Robert Hertz (1913) dans son étude sur saint Besse qu'il rattache à un antique culte des pierres. Sous les traits de Ghāzī Miyāñ se cache une source d'eau bienfaitrice, contenue dans un bassin dédié au dieu hindou Soleil qui lui sert de sépulture.

Les récits de la vie de ce martyr opposent les hautes castes réfractaires à son culte aux basses castes adeptes de celui-ci, comme les « deux traditions, l'une savante et édifiante, l'autre naïve et poétique » (Hertz, 1913, p. 152) de saint Besse opposent les gens de la montagne aux gens de la plaine. Néanmoins ici, en Uttar Pradesh où se situe le tombeau, tous, tenants du culte et opposants, appartiennent de par leurs activités au monde rural (même les citadins). La Shining India est encore loin d'atteindre cette région, dont les habitants disent d'eux-mêmes être « arriérés ». Ici la culture des patrons (grands propriétaires terriens) et des savants (docteurs de la loi musulmane, juges, professeurs, instituteurs, etc.) de castes nobles s'oppose à celle de leurs clients de bas statut (artisans, paysans, bouviers, castes de service, etc.), notamment en ce qui concerne le dogme religieux (musulman et hindou puisque les dévots de Ghāzī Miyāñ professent ces deux religions) et le culte des saints, ce que soulignent les divergences entre les deux traditions.

Toutefois, celles-ci s'accordent pour faire de ce martyr, dont on ne trouve trace dans l'histoire, un jeune héros se sacrifiant sur le champ de bataille à l'aube de sa vie (dix-neuf ans) encore vierge et célibataire, mais aussi l'un des plus anciens saints ( $\mathrm{XI}^{\mathrm{e}}$ siècle) et des plus populaires de l'Inde du Nord, dont le tombeau (situé dans la ville de Bahraich) attire des millions d'Indiens de toutes confessions, originaires du nord du sous-continent indo-pakistanais. Nous ne nous attachons pas dans cet article à analyser ce qui unit, distingue et oppose les deux traditions ${ }^{2}$. Dans leurs grandes lignes, elles ne s'écartent pas de celles des premiers saints indo-musulmans, qui mettent en scène les batailles qu' ils durent mener contre des souverains hindous, des déités locales et des ascètes yogi pour faire souche en Inde ${ }^{3}$. C'est en triomphant du démon Palihār et des Pañc Deo et en combattant au prix de sa vie le tyrannique roi hindou de Bahraich, que Ghāzī Miyāñ a conquis sa réputation de défenseur des paysans hindous opprimés.

2. Nous avons consacré à ce sujet deux articles, voir D. ORTis, 2016 a et 2016 b (ce dernier proposant une bibliographie complète sur ce saint).

3. Voir pour l'Inde du Nord le cas de Mu'īnal-dīn Chishtī de Ajmer (Currie, 1989, p. 66-96) et pour l'Inde du Sud celui de Bābā Budhan au Karnataka (Sikand, 2004, p. 170-171). 
Puisque les récits de vie ne sont pas indispensables à la pratique du culte aux saints, nous nous proposons d'étudier leur place dans le culte de ce héros et la place de leurs auteurs dans la société locale. Deux pratiques rituelles associées à leur audition seront donc notre fil conducteur : la visite propitiatoire pour la légende des desservants de son tombeau ; la célébration de son mariage pour les ballades des musiciens dafälì. Nous terminerons par les récits qui cherchent à défaire ce culte.

\section{Le rite de la visite propitiatoire et la légende des desservants}

Pour obtenir les faveurs de Ghāzī Miyāñ, le dévot doit « faire une visite propitiatoire » (ziyārat karnā $)^{4}$ à son sanctuaire, impliquant une série d'actes médiatisée par un desservant pour en accroître les effets : salutation, prières, offrandes, incorporation de la puissance du saint, circumambulation. Que ce soit simplement pour le saluer ou pour quémander son intercession, le dévot ne peut faire l'économie de cette visite, qui lui permet d'initier et de cultiver une relation avec le martyr et en définitive d'obtenir de lui ce qu'il désire. Cette visite est à la fois commune à tous les saints musulmans et spécifique dans ses modalités à chacun d'eux ${ }^{5}$.

Celle accomplie pour Ghāzī Miyāñ implique de traverser en partie le site de son martyre. Ce champ de bataille s'étend sur des centaines de kilomètres et est vu aujourd'hui comme une nécropole recouverte par la poussière du temps. Puis entrer dans son sanctuaire exige de circuler dans un espace architectural conçu comme un campement militaire nommé les quatre limites, dans lequel siègent deux forts enchâssés l'un dans l'autre contenant le mausolée de Ghāzī Miyāñ, luimême marquant l'emplacement exact du martyre. Mais la visite ne s'arrête pas là.

Elle demande ensuite d'aller saluer les membres de sa cour représentés par vingt tombes. Le visiteur doit s'arrêter sur chacune d'elles pour saluer celui qui y repose et lui faire de menues offrandes. Le sens de la visite est défini précisément par l'organisation des lieux : on salue en premier son maitre d'armes, puis ses deux serviteurs, sa chienne, sa jument, le porte-étendard de son armée, le trésor des mar-

\footnotetext{
4. Les termes vernaculaires cités appartiennent à l'hindoustani (bindustānī), qui désigne l'ensemble linguistique formé par l'ourdou et le hindi (langues séparées depuis la partition de 1947). Ces deux langues reposent sur un fonds linguistique commun et divergent par leur écriture (en caractère nasta'līq pour l'ourdou, en caractère devanāgarī pour l'hindi) et leur vocabulaire savant (d'origine arabo-persane pour l'ourdou, sanskrite pour le hindi).

5. L'ouvrage de H. Chambert-Loir et C. Guillot (1995) est là aussi un outil précieux offrant un aperçu de la diversité des pèlerinages sur les tombes des saints ; voir les six articles consacrés à l'Asie du Sud (p. 167-234).
} 
tyrs rassemblant ses soldats, son épouse post-martyre Zohrā Bībī et ses deux frères, son ami, son oncle maternel, les Cinq Saints et leurs deux gardes du corps (des lutteurs) ainsi que leur boucle d'oreille et enfin sa nourrice.

À proximité de chacune de ces tombes, une pancarte indique le nom de la personne inhumée, en caractère nasta'līq et devanāgarì. Si l'on se contente de ces noms, on est renvoyé à des fonctions (maître, serviteur, lutteur, nourrice, porte-étendard) ou à des statuts (caste des descendants de la famille de Mahomet, sieur, dame). Ces indications ne prennent vraiment sens pour le visiteur que dans la bouche des desservants qui, par leur légende, donnent vie à cette cour et tissent les relations entre ses membres et Ghāzī Miyāñ.

En effet, la fréquentation d'un sanctuaire, même assidue, n'implique d'être au fait ni de la légende de son saint ni de l'histoire du lieu. Savoir par ouï-dire que Ghāzī Miyāñ est un grand thaumaturge, spécialisé dans la résolution des problèmes de la vie courante (obtention d'un diplôme, d'un travail, d'un bon parti, etc.), des problèmes d'infertilité (son premier miracle fut de donner des garçons à une hindoue stérile), dans la guérison de la cécité et des maladies de peau (la lèpre et la leucodermie) et qu'il assure de bonnes récoltes en faisant venir la pluie, suffit pour se rendre en pèlerinage à son sanctuaire et y accomplir le rite de la visite ${ }^{6}$. Les curieux voulant en connaître plus, doivent faire l'achat d'un livret vendu au sanctuaire, mais le plus souvent, les visiteurs étant encore largement analphabètes, ils s'en remettent aux récits que les desservants délivrent oralement. Il suffit donc au cours de la visite de demander au desservant assis contre la tombe de faire le récit de la vie de Ghāzī Miyāñ, en échange ou non d'une petite rétribution. Il est habituel que les personnalités importantes bénéficient d'une visite guidée, au cours de laquelle un desservant conte la légende du martyr.

Les desservants ont deux façons de raconter ce qu'ils appellent l' « histoire » (itihās) de Ghāzī Miyāñ, comme nous en avons fait l'expérience. Il est à noter que si nos entretiens avec les desservants attiraient toujours des badauds ils ne restaient jamais bien longtemps, sauf la fois où nous demandâmes que l'on nous raconte la légende. Ce jour-là, les nombreux visiteurs installés autour de nous écoutèrent jusqu'au bout et en profitèrent pour poser des questions. Le desservant peut donc

6. C. Bellamy tire à ce propos les mêmes conclusions que nous de son étude du sanctuaire de Husain Tekrī (à Jaora, Madhya Pradesh) : « The details of the stories are not known by most pilgrims who visit the shrine [...] For most pilgrims, even long-term residents, it is enough to know that the saints are present in the shrines: the origin stories of Husain Tekri are not, in other words, significant components of the authority and legitimacy of the present-day site, which centers on the notion that the rauzas [tombeau] are places that channel the power of the saints to the pilgrims » (2011, p. 45). 
prendre pour point de départ à son récit le héros lui-même : il commence par les circonstances de sa naissance, puis déploie avec plus ou moins de détails les différents épisodes de sa vie pour terminer par son second miracle, le mariage que le héros organisa lui-même après son martyre. Il peut aussi raconter cette histoire de façon fragmentaire, en prenant pour fil conducteur un membre de la cour. Généralement, ce procédé fait suite à une question sur l'identité d'un d'entre eux ou sur les raisons de sa présence en ce lieu. Même si les desservants parlent a priori d'un autre personnage, ils font là aussi le récit de la vie de Ghāzī Miyāñ, car ce que l'on apprend ainsi concerne essentiellement celui-ci.

Pour exemplifier notre propos, prenons le récit entendu sur sa nourrice : « La mère de Ghāzī Miyāñ mourut en couche et son père appela une nourrice pour prendre soin de son fils. Elle lui donna le sein, le berça et l'éleva. Elle prit soin aussi de sa maison et de son linge, comme une servante. Durant la bataille, elle souleva l'épée et mourut en martyr au combat. Grâce à son service auprès de Ghāzī Miyāñn, elle est devenue comme une mère pour lui et a acquis un statut élevé ». Rien de plus n'est dit sur cette femme. Son nom, Nainā Dāyah Sāhibā, n'apporte pas d'élément personnel mais confirme sa mission de « nourrice » (dāyah) et son appartenance à la caste des Barbiers (naina « femme de barbiers »), qui renvoient l'un à l'autre puisque dans cette société la femme barbier est traditionnellement sage-femme et nourrice.

La narration par fragments offre néanmoins plus de détails que la narration biographique. Par l'intermédiaire de la nourrice, les desservants déploient l'enfance de Ghāzī Miyāñ qu'ils peuvent compléter à leur guise avec le récit sur son maître d'armes : « Il l'initia dès son plus jeune âge à l'art de la guerre : maniement de l'épée, tir à l'arc et apprentissage du Coran $\gg$. Si ces deux personnages permettent aux desservants d'évoquer l'enfance du héros, ils mettent en relief par là-même son appartenance à la classe des nobles guerriers et justifient l'organisation du sanctuaire, dans laquelle il a le statut de « maître de maison » (jajmān) et donc le droit d'être le patron de famille de castes de service.

Ces récits montrent de plus que ces personnages secondaires ne sont pas isolés et entretiennent entre eux des relations, ainsi : « Rajab Sālār est le frère de Nainā Dāyah, c'est pourquoi il est l'oncle maternel de Ghāzī Miyāñ. Son vrai oncle maternel est Mahmūd Ghaznavī $\gg^{7}$. La relation frère-sœur et la parenté de lait expliquent donc le lien entre Rajab Sālār et Ghāzī Miyāñ. Comme pour sa sœur et le maître d'armes on sait peu sur lui : il fut l'ami de Ghāzī Miyāñ et le

7. Mahmūd Ghaznavī (r. 997-1030) est un des plus célèbres souverains du monde musulman médiéval qui durant son règne pilla l'Inde du Nord. 
général de son armée. Mais à son tour ce personnage en fait entrer d'autres dans le récit, puisqu'il est précisé qu'il avait sous ses ordres les oncles paternels du héros (enterrés dans la jungle de Bahraich). Tout en enrichissant le récit de la vie de Ghāzī Miyāñn, à travers ces personnages secondaires ce sont les fondements de la société indienne qui sont dépliés - relation de parenté et de castes -, montrant par là-même que le saint fait aussi société dans son sanctuaire.

Connaître les légendes des desservants permet de comprendre à qui on adresse ses salutations et de choisir parmi les membres de la cour celui dont on se sent le plus proche (par la caste, la fonction, le genre, le goût), pour lui confier ses peines et ses espoirs et escompter que par son intersession on sera entendu du martyr qui, par sa puissance et son statut, peut sembler inaccessible. Connaître la légende des desservants, c'est encore pouvoir pénétrer les secrets du lieu. Ainsi, la nécropole n'est visible qu'à ceux qui connaissent le récit de la visite du sultan Fīrūz Shāh Tuğlūq (r. 1351-1387) venu remercier le martyr de lui avoir apporté la victoire à la guerre (troisième miracle) : « Le sultan voyagea à cheval jusqu’à la rivière Ghaghra, où il rencontra le saint Mīr Mah Shāh envoyé par Ghāzī Miyāñ pour l'assister dans sa visite. Celui-ci expliqua au roi qu'ils devaient poursuivre leur chemin à la seule force de leur gros orteil pour ne pas souiller le sol jonché de cadavres, qu'il dévoila au roi en le coiffant de son chapeau $\gg$. De même, seuls ceux qui connaissent l'emplacement de la tombe du maître d'armes, sous la marche du seuil du tombeau de Ghāzī Miyāñ, savent qu'en entrant ils le foulent de leurs pieds selon ses dernières volontés. Ou encore que la tombe de son ami n'est en fait qu'un cénotaphe et que son corps est inhumé près de Ghāzī Miyāñ, en témoignage de leur affection réciproque.

Ce corpus de récits de vie, tout succinct qu'il soit, rend intelligible la visite propitiatoire en associant chacune de ces tombes à l'expérience d'une vie singulière auprès de Ghāzī Miyāñ, qui justifie sa présence en ces lieux et les dévotions dues, auxquelles le visiteur ne peut se soustraire sans risquer de provoquer le mécontentement du martyr. Tous les personnages en ont besoin mais certains plus encore, comme la jument et la chienne. Selon les prescriptions islamiques, l'inhumation des animaux est interdite, notamment parce qu'elle est source d'impureté, justification qui prend une résonnance toute particulière dans la société indienne, où la valeur de l'opposition hiérarchique du pur et de l'impur fonde le système des castes ${ }^{8}$. Or, dans la légende des desservants, l'édification de leurs sépultures semble être une évidence : « La jument et la chienne sont martyrs. Elles ont veillé 
sur l'intégrité du corps de Ghāzī Miyāñ jusqu'à mourir de faim, en cachant son cadavre dans un buisson, afin que ses ennemis ne puissent le retrouver. Elles ont donc leur place en ce lieu ».

En définitive, tous les personnages jouant un rôle majeur dans les récits des desservants sont enterrés auprès de Ghāzī Miyāñ, à moins qu'il ne soit plus juste de dire que tous ceux qui sont aujourd'hui auprès de lui sont les principaux personnages des récits des desservants. Quoi qu'il en soit, ces derniers sont les seuls à construire leurs récits en s'appuyant sur la configuration du lieu de culte. Quels que soient leurs procédés (biographique ou par fragments), ils déploient narrativement l'espace du sanctuaire qui, en contrepartie, donne chair à leur récit, puisque la permanence de ces tombes censées datées du XI $\mathrm{XI}^{\mathrm{e}}$ siècle, c'est-à-dire trois siècles avant l'installation des musulmans dans la région, est bien pour les dévots la preuve que ces personnages ont effectivement existé. Ils continuent de vivre aujourd'hui grâce au culte de leur tombe et aux récits de leurs hauts faits. Ces récits concernent autant le martyr que la fondation de son sanctuaire et l'instauration de son culte.

Qui sont les desservants ? - Ils affirment eux-mêmes descendre des deux serviteurs de Ghāzī Miyāñ, les seuls survivants de sa caravane et ses uniques héritiers (le martyr est fils unique et vierge). Si l'on en croit leur légende, qui institue l'hérédité de leur fonction - le service de Ghāzī Miyāñ et de son tombeau -, depuis plus de neuf cent cinquante ans ceux-ci se trouvent sous leur surveillance.

Alors que Ghāzī Miyāñ mène la bataille [contre le roi hindou de Bahraich], son père tombe malade à Satrikh. Il ne peut s'y rendre et envoie ses deux serviteurs à son chevet. Son père meurt quelque temps plus tard ${ }^{9}$. Devenu martyr, Ghāzī Miyāñ rappelle par un rêve ${ }^{10}$ ses deux serviteurs pour qu'ils continuent de le servir. Les deux frères s'installent ici même. Les générations s'accroissent doucement. Les hommes nettoient la jungle, la cultivent et la population grandit. De cette façon, une organisation se développe ici. [...] Maintenant il y a environ mille desservants.

Selon leur légende, les desservants ont fait passer le site du tombeau du monde sauvage (la jungle) au monde civilisé (l'agriculture). La population de Bahraich leur attribue d'ailleurs la fondation du village Singha Parasi, site du sanctuaire,

9. Sa tombe se trouve à Satrikh (district de Bara Banki UP) à une centaine de kilomètres de Bahraich.

10. Le rêve est le moyen utilisé par les saints pour communiquer avec le commun des mortels. 
154 Des vies extraordinaires : les territoires du récit $-\mathrm{n}^{\circ} 79$

aujourd'hui intégré aux limites administratives de Bahraich. Un certain nombre d'entre eux exercent toujours les deux fonctions : l'agriculture et le service du tombeau, dont la tâche la plus importante est la production de l'eau du bain de la tombe, véhicule de la thaumaturgie de Ghāzī Miyāñ, que ses dévots boivent ou dans laquelle ils se baignent. En s'incluant dans la légende qu'eux-mêmes diffusent, les desservants légitiment bien sûr leur position mais également l'organisation spécifique de ce sanctuaire qui repose sur le système jajmānī (liant héréditairement un maître de maison à des castes de service), dans lequel les desservants sont les premiers à servir le maître de maison qu'est Ghāzī Miyāñ. Si ce mode d'organisation est exceptionnel pour un sanctuaire musulman, il régit la société locale. Et c'est la principale qualité des narrations des desservants de renvoyer par différents moyens à la localité, en mettant en valeur la méta-caste locale yādava ${ }^{11}$, qui représentent une part importante des dévots de Ghāzī Miyāñ, et ont leur place dans sa cour à travers les lutteurs (la lutte est une activité qu'affectionnent les membres de ces castes) et leur boucle d'oreille (portée par les hommes yādava). Le premier miracle du martyr fut en sus accompli en faveur d'une femme abìr. Car leur légende est aussi un discours visant à promouvoir ce qu' ils nomment « l'ordre socio-cosmique du fort de l'ici du Bābā » (bābā ke yahāñ kā qil'a kā dharma $)^{12}$ et qu'ils défendent contre ceux qui cherchent à le dénigrer en le qualifiant de pratique superstitieuse des basses castes ou contre ceux qui cherchent à l'islamiser.

Si nous voulons comprendre comment s'élabore le culte aux saints, il faut accorder dans nos études une plus grande place aux desservants des tombeaux. Animés par le désir et le besoin de voir le culte du saint qu'ils servent se perpétuer et s'étendre, ils doivent par leurs récits attirer les dévots et les retenir. Pour ce faire, il est indéniable, même si nous ne pouvons en apporter ici une preuve formelle, qu'ils adaptent leurs légendes aux évolutions sociales et contribuent à faire des saints des personnages toujours contemporains. Grâce au développement des communications, ils exportent de plus en plus loin leur récit. L'un de nos principaux interlocuteurs était un desservant habile en fabrication d'amulettes, qui officiait dans toute l'Inde du Nord. Lors de notre séjour, il se rendit à Bhopal et

11. Appellation réunissant trois castes pastorales et martiales (Ahīr, Gopi [ou Gopa] et Goala), parmi lesquelles les rois recrutaient leurs soldats et qui sont aujourd'hui autant pasteurs que cultivateurs. Les Yādava représentent la troisième force démographique de l'U. P. Ils appartiennent au varna des « serviteurs » (Ś̈̈dra) situé très bas dans la hiérarchie et sont répertoriés par l'État indien comme « classes arriérées autres 》 (Other Backward Classes, OBC). Au sujet des Yãdava voir C. Servan-Schreiber (1999, p. 16 et 109-112) et W. R. Pinch (1996).

12. Bābā est un terme affectueux pour désigner un saint. 
nous dit qu'il ne manquerait pas de rappeler qu'un roi de cette ville naguère guéri de la lèpre grâce à Ghāzī Miyāñ lui offrit en retour un grand porche pour son sanctuaire. Il nous précisa qu'il profitait toujours de ses voyages pour rallier de nouveaux adeptes en faisant le récit des miracles de son Bābā, dont il tirait son don ${ }^{13}$.

Le rôle des desservants, tels que ceux qui œuvrent dans ce sanctuaire, est généralement tu dans les ouvrages ethnologiques et historiques, alors qu'ils sont au cœur de la relation saint-dévots et peuvent, comme ici, en fixer librement les règles. Il est vrai que sous le terme $x \bar{a} d i m$ (transcrit aussi khādim), on trouve dans la littérature différents types de desservants (custodians ou caretakers en anglais). On entend généralement par ce terme, les héritiers des saints (soit par les liens du sang nommés gaddī nasìin, soit spirituellement sajjādah nasiinn), ou encore les employés des « fondations pieuses » (awqaf), sorte de fonctionnariat qui s'est mis progressivement en place depuis l'administration britannique ${ }^{14}$. Ces deux types de desservants gèrent aujourd'hui ensemble (en bonne entente ou de façon conflictuelle) la plupart des sanctuaires ${ }^{15}$. On dispose maintenant d'une littérature conséquente analysant au cours du temps leur puissance politique et économique et leurs relations avec le pouvoir temporel. Ce qui frappe dans ces études de cas est la grande diversité des modes d'organisation des sanctuaires ${ }^{16}$. Toutefois, les desservants du type de ceux que l'on trouve attachés au culte de Ghāzī Miyāñ (descendants de ses deux serviteurs) sont eux laissés de côté par cette littérature, comme le dit Marc Gaborieau (2003 b, p. xiii) : «The 'low caste' fakirs and the

13. Toutefois, à la différence d'autres sanctuaires thérapeutiques, les desservants de Ghāzī Miyāñ n'accomplissent aucune pratique curative ; pour une étude approfondie sur le rôle du culte des saints dans les pratiques curatives, voir F. Speziale (2010).

14. Voir à ce sujet K. Rashid (1978) et G. C. Kozlowski (1985).

15. Voir E. A. Mann (2003), pour un exemple situé dans la même région.

16. Pour poursuivre avec le sanctuaire de Mu'īnal-dīn Chishtī, voir l'étude de S. L. H. Moini (2003) et de P. M. Currie (1989, p. 141-184) qui distingue de façon détaillée les différents acteurs faisant fonctionner ce lieu; voir U. FALASCH (2016) pour le sanctuaire de Zinda Śāh Madār, autre grand saint de l'U. P. en relation avec Ghāzī Miyāñ. 
menials [ainsi les nomme-t-il] are usually neglected in the descriptions of the shrines: in constrast with the caretakers $\gg{ }^{17}$.

Notre manque d'intérêt tient certainement au fait que ces hommes de bas statut, peu éduqués, sont mésestimés par les autres types de desservants et plus globalement par les hautes castes musulmanes, qui les considèrent au mieux comme de simples balayeurs (les desservants de Ghāzī Miyāñ sont catalogués comme sweepers par les membres de l'administration du sanctuaire, tous de hautes castes), au pire comme des profiteurs. Le desservant est souvent décrit comme un pauvre qui s'est installé à côté d'une tombe et l'a recouverte d'une étoffe ${ }^{18}$ pour soutirer de l'argent à une population crédule à la première fable entendue. Cette critique est a contrario la preuve de leur importance dans la fabrique du culte aux saints, comme intermédiaire entre le saint et ses dévots, comme interprète de son culte et de sa légende. Le travail de Carla Bellamy montre pour sa part que cette dernière fonction peut être assumée par les pèlerins eux-mêmes, qui échangent entre eux oralement leurs connaissances sur les récits de vie des saints et sur les pratiques prophylactiques et curatives. Ces faits, que révèlent peu à peu un nombre croissant de monographies de sanctuaires menées par des ethnologues, nous conduisent à reconsidérer le rôle des desservants de hautes castes (gaddī naśīn, sajjädah naśīn), des employés des fondations pieuses et des hagiographies privilégiés par les historiens travaillant à partir de sources écrites. Ces nouvelles ethnographies relativisent l'importance des textes savants dans la pratique cultuelle et mettent en relief les multiples voies/voix que prend la diffusion des légendes locales dans le cadre du culte.

Ainsi, aux côtés des desservants de Ghāzī Miyāñ, œuvrent les musiciens dafālī, eux aussi comme propagateurs de la légende et officiants rituels, mais cette fois lors des fêtes.

17. Dans un article sur la signification historique des tombes à Java (en Indonésie), J. J. Fox insiste aussi sur l'importance cruciale des gardiens des tombes de saints dans l'élaboration, la réinvention de l'histoire passée : «I argue in this chapter that jurukunci [les gardiens en question] throughout Java play an extraordinarily important - though of - the overlooked [c'est moi qui souligne] - role in interpreting and disseminating views of Java's past.[...] At some tombs jurukunci provide, sometimes only on request, their own stenciled accounts $\gg(2002$, p. 161).

18. L'étoffe, en tant qu'offrande la plus courante, est le premier élément indiquant qu'il peut s'agir de la tombe d'un saint. 


\section{Les fêtes en l'honneur de Ghāzī Miyāñ et les ballades des Dafālī}

Les Dafālī interviennent pour le Cinquième jour de printemps et la Foire de mai-juin. Leur première fonction est de diffuser auprès des dévots les dates de ces deux fêtes, que leur communique l'administration du sanctuaire. Ils se rendent chez eux et, en interprétant leur répertoire, leur rappellent le bien-fondé de leur participation s'ils veulent demeurer sous la bénédiction de Ghāzī Miyāñ. Leur seconde fonction est de mener chez ce dernier les dévots et leurs offrandes en cortège cérémoniel.

Le Cinquième jour de printemps est, à la fois, une fête des prémices offerte à Ghāzī Miyāñ en tant que garant de la récolte d'hiver, et une fête des augures lors de laquelle la récolte d'été est placée sous sa protection. Pour ces deux séquences, les Dafālī battant tambour conduisent les cortèges au tombeau afin qu'ils offrent les grains voués au martyr. Les dévots qui ne peuvent venir font parvenir leurs contributions par leur intermédiaire.

Les Dafālì officient également à cette occasion pour les agents du sanctuaire (les desservants et les membres de l'administration), car ceux-ci sont les premiers bénéficiaires des revenus des terres agricoles appartenant à Ghāzī Miyāñ. Réunis dans le bureau de l'administration, ils écoutent la prestation du plus âgé des Dafālī (représentant de sa caste) qui interprète plusieurs ballades de son choix, sur les paniers contenant les prémices et les augures. Puis tout le monde se rend en procession au tombeau au rythme des tambours, pour offrir leur contenu au martyr.

La fête se clôt par l'annonce de la date du mariage de Ghāzī Miyāñ, célébré lors de la Foire de mai-juin. Les Dafālī se font alors enregistrer auprès de l'administration pour recevoir des aides financières et logistiques en vue de la préparation des cortèges cérémoniels de cette fête, qui célèbre le mariage de Ghāzī Miyāñ avec Zohra Bībī, une jeune hindoue que notre héros a rencontrée lors de sa traversée de l'Inde du Nord.

Alors que deux épisodes de la biographie des desservants sont consacrés à ce thème - le premier narre leur rencontre, le second les modalités particulières de la célébration du mariage à la suite du martyre - les ballades des Dafālī ne disent mot de ce mariage avec Zohra Bībī. Au contraire, celles-ci racontent que Ghāzī Miyāñ est né sous l'imprécation de mourir non marié le jour de ses noces, proférée par le saint Zinda Sāh Madār ${ }^{19}$ auquel les musiciens attribuent sa naissance miraculeuse. Si la prophétie se réalise dans un premier temps, puisque dans les deux traditions

19. Zinda Śāh Madār (m. 1440) est l'initiateur de la confrérie soufie Madārī. Sa tombe est située à Mākānpur, à deux cents kilomètres environ de Bahraich. 
Ghāzī Miyāñ est célibataire au moment de son martyre, elle ne l'empêche pas de se marier post-martyre, et aux Dafālī d'être officiant rituel de ce mariage et donc d'inverser la malédiction qu'ils colportent. Les deux traditions agissent ici de façon à renforcer l'aspect prodigieux de ce mariage, considéré par tous comme le plus grand miracle de ce héros, pour les raisons suivantes : il l'a organisé lui-même après son martyre et il est réitéré chaque année par ses dévots. Les desservants achèvent d'ailleurs leurs récits sur l'idée qu'il en sera ainsi jusqu'au Jugement Dernier et que la vision des cortèges cérémoniels se rendant au mariage est la première cause de la propagation du culte. Dans cette société sans église, la sainteté est reconnue au nombre de dévots, que la capacité d'accueil de leur sanctuaire corrobore. L'accomplissement de ce mariage et sa répétition annuelle sont donc la preuve incontestable que la puissance de Ghāzī Miyāñ surpasse celle de Zinda Śāh Madār, l'autre grand saint de la région.

Durant la Foire, les Dafālī organisent trois processions. La première se nomme « les foires » (mele) et accomplit le pèlerinage (aller-retour); ils y remplissent trois fonctions. Les « porteurs de fouet » (kore bārdār) font office de gardes et de guides : ils vont en avant pour tracer le chemin et diriger la procession en jouant de leur fouet comme d'une cloche, disent-ils, pour attraper et capter le cortège. Ils sont suivis par « celui tenant » (gīrdār) le drapeau rouge de Ghāzī Miyāñ, qui précède les foires chargées de leurs offrandes et du nécessaire pour camper dans le sanctuaire. Celles-ci avancent au rythme des « faiseurs de bruit » (dhümdār) qui frappent leur tambour et chantent les ballades. Le voyage se fait en partie ou entièrement à pied, ce qui implique pour certains de faire plusieurs centaines de kilomètres. Ces cortèges, colorés et bruyants, attirent les curieux qui, les voyant passer, entendent les appels à l'aide lancés à Ghāzī Miyāñ et quelques bribes des ballades.

La deuxième procession se rend au tombeau de Ghāzī Miyāñ pour lui offrir une «bannière » (niśān), faite d'un grand patchwork fixé à une longue perche en bambou dont l'extrémité est ornée d'une queue de vache ou d'un collier de pièces de monnaie. La procession danse un long moment avec sa bannière au rythme des tambours des Dafālī, avant d'essayer de toucher par son intermédiaire le pinacle du tombeau, en prononçant des vœux et en promettant de revenir l'an prochain s'ils sont exaucés. Les bannières sont présentées plusieurs années de suite jusqu'à exaucement des vœux, dont elles sont le « symbole », la « preuve », la « trace », ainsi que l'indique la polysémie du terme niśān faisant référence à toutes ces notions. Les premières bannières, selon une ballade dafâlì, furent celles de pauvres habitants de la région aidés par Ghāzī Miyāñ qui voulurent rejoindre son armée pour se battre à ses côtés contre le roi de Bahraich. Mais lorsqu'ils arrivèrent, ils 
ne trouvèrent que la tombe du martyr et décidèrent de laisser leurs bannières tout autour.

Enfin, les Dafālī mènent les « processions de mariage » (bārāt $)$ au tombeau de Ghāzī Miyāñ. A l'inverse de la célébration du mariage du commun des mortels, pour lequel c'est le jeune marié qui se déplace chez le père de la jeune mariée, lesdits cortèges viennent offrir à Ghāzī Miyāñ Zohra Bībī, représentée par un palanquin contenant sa dot. Le don de l'épouse est accompli par les Dafālī conduisant la procession auprès du scribe du sanctuaire qui l'enregistre. Le matin même, ils avaient consacré les bannières, les biens de la dot et le repas de noces préparé pour Ghāzī Miyāñ et sa cour, en récitant sur eux la première sourate du Coran (l'« Ouverture », fätehah).

L'association des Dafālī (4000 membres) accomplit aussi la visite propitiatoire en procession. Les porteurs de fouet ouvrent la marche, suivis par les porteurs du drapeau rouge de Ghāzī Miyāñ et d'une banderole sur laquelle se trouvent toutes les informations concernant le sanctuaire, puis viennent les musiciens tambourinant et enfin les femmes dafälī portant sur leur tête les offrandes dédiées au martyr. La procession avance lentement en s'arrêtant régulièrement pour laisser les porteurs de fouet danser et faire exploser des pétards. Elle est accueillie à l'entrée du fort par le discours d'un desservant. Puis les tambourinaires chantent quelques ballades rétribuées par le public présent. La procession se rend enfin au tombeau, pour offrir sa musique et ses offrandes à Ghāzī Miyāñ.

Comme nous l'avons vu, au cours de toutes ces processions, les Dafālì frappent tambour et chantent, toutefois leurs vers se perdent dans le brouhaha car, comme pour toutes les processions de bon augure en Inde, il est surtout question de faire du bruit pour attirer l'attention des humains et des êtres divins. Leur poésie chantée n'est vraiment appréciable qu'à l'occasion du concert qu'ils donnent pour montrer, selon leurs dires, leur amour de Ghāzī Miyāñ, en diffusant la grandeur de son âme à travers le récit de sa vie exemplaire. A l'inverse des processions, la voix est au premier plan soutenue par le tambour frappé de façon à ponctuer l'action dramatique de la narration. Les Dafālī se produisent alors en duo (un chanteur et un tambourinaire), debout encerclé par les pèlerins assis autour d'eux. Le chanteur prononce d'abord le vers a cappella, qui est ensuite chanté par le duo accompagné par le tambour frappé pianissimo ou fortissimo suivant l'émotion que le duo veut produire sur le public. Quand l'auditoire est effectivement ému, suite à l'audition d'un vers particulièrement beau et poignant, il se lève pour gratifier le duo. Ce genre de prestation a aussi lieu hors des périodes festives les jours d'affluence.

Ces poèmes narratifs sont composés par des poètes (dafälī ou autres), en vers rimés ou libres, dans différentes langues vernaculaires de la région (avadhi, hindi, ourdou et bhojpuri, cette dernière étant la plus prisée car elle est considérée 
comme la plus poétique de toutes). Ils content la vie de Ghāzī Miyāñ sous forme d'épisodes, dont les plus célèbres sont dans l'ordre de leur narration : la naissance du saint guerrier, le démon Palihar, le lai de sainte Amina, la quête de Mare Lilli (nom de la jument du martyr), la chute du roi Banar et le mariage du saint guer$\operatorname{rier}^{20}$. Chaque épisode comprend plusieurs ballades ${ }^{21}$. Lors des performances, tous les épisodes et toutes les ballades ne sont pas obligatoirement interprétés. Suivant le temps disponible, le désir du Dafālī ou du public, certains peuvent être omis. Les Dafālī composent aussi des louanges, soulignant la grandeur du héros qui jusqu'à aujourd'hui sait donner asile, consoler et exaucer les plus faibles, les stériles et les malades. Selon l'historien des subaltern studies Shahid Amin (2005, p. 281 et p. 283), ce répertoire est attesté depuis la fin du XIII ${ }^{\mathrm{e}}$ siècle ${ }^{22}$.

Qui sont les Dafālī ? Une sous-caste de la caste des « musiciens professionnels » (mīrāsī) jouant du tambour nommé dāf. Ces musiciens itinérants sont connus dans la littérature ethnographique coloniale pour avoir été les prêtres des « adeptes du culte des Cinq Saints » (pāñ $\tilde{c} \bar{i} r y \bar{a})$, dont la série varie d'une localité à l'autre mais dans laquelle Ghāzī Miyāñ occupe toujours la première place $^{23}$. L'origine de ce quintette demeure obscure et il semble aujourd'hui avoir été délaissé au profit du culte unique de Ghāzī Miyāñ. Toutefois, l'un comme l'autre ont la particularité de rassembler hindous et musulmans de basses castes autour d'un culte dédié à des entités musulmanes et hindoues, comme l'illustre le récit fragmentaire des desservants concernant les Cinq Saints de la cour de Ghāzī Miyāño, cinq géants hindous envoyés par le roi de Bahraich pour le tuer :

Ils arrivent dans un orage et une tempête de poussière dans le but d'emporter Ghāzī Miyāñ dans leur souffle. Celui-ci est en train de jeûner. Il leur demande de lui laisser le temps de rompre son

20. Dans cet épisode le nom de l'épouse n'est pas précisé.

21. D'une à dix ballades selon nos informations, mais rien n'indique qu'il n'y ait pas plus de ballades dans chaque épisode, ni qu'il n'existe pas plus d'épisodes.

22. Toutefois, il est bien difficile de dater ce répertoire, dont on ne connaît pas l'étendue du corpus, et son apparition dans les rites, pour une société privilégiant l'oral à l'écrit. R. C. Temple (1884) et R. Greeven (2010) sont les premiers à avoir publié quelques-unes de ces ballades. Le matériel recueilli par S. Amin ne diffère guère des compilations des ethnographes britanniques de la fin du $\mathrm{XIX}^{\mathrm{e}}$ siècle. Celles enregistrées à Bahraich sont inédites. Ces ballades ne sont pas exceptionnelles et s'inscrivent dans la riche tradition orale indienne (voir à ce sujet C. SERVAn-SChreIber 1999, p. 7).

23. Voir à leur sujet W. Crooke (1968, vol. I, p. 206), J. Briggs (1953, p. 489), G. A. Herklots (1972, p. 67), K. G. v. SCHWERIN (1981, p. 152-153). 
jeûne et de faire sa prière avant de se battre. Mais les géants ont faim et décident de le manger. Ghāzī Miyāñ leur propose son pain et son sel. Alors qu'ils s'avancent, il les fait pénétrer dans son pot. Puis au moment de rompre son jeûne, il met dans le pot le pain et le sel qui se dirigent d'eux-mêmes dans la bouche des géants, qui ont alors foi en lui et restent avec lui.

Cette sous-caste est aussi connue pour chanter dans les cérémonies de mariage et pour écrire des chansons de guerre, deux thématiques majeures de la geste de Ghāzī Miyāñ et que l'on retrouve dans la double origine dont ils se prévalent. En effet, ils affirment descendre des tambourinaires ayant suivi Mahomet durant l'Hégire ${ }^{24} \mathrm{~d}$ 'une part, et avoir été chargé par celui-ci de la fonction de musiciens de mariage, puis par Ghāzī Miyāñ de celle de porte-drapeau de son armée d'autre part. Selon les desservants, les Dafālī étaient des soldats de l'armée de Ghāzī Miyāñ et ne commencèrent à composer des chansons et à jouer de la musique pour les mariages qu'après le martyre, ce qui expliquerait que leurs fonctions (kore bārdār, gìrdār et dhümdār) se retrouvent dans l'armée.

Comment passe-t-on de la guerre au mariage ? Ou du mariage à la guerre ? Comme ils le chantent dans l'épisode du mariage du saint guerrier :

Jouez la musique de mariage, dumarā.

Abandonnez la musique de mariage.

Jouez la musique de guerre.

Les Dafālī, comme leur saint, sont liés aux domaines de la guerre et du mariage. Cette association guerre/mariage est un des traits de la tradition épique indienne ; qu'elle soit savante ou populaire, écrite ou orale, elle magnifie les exploits de ses héros, dont le rôle est d'étendre le territoire et de le protéger aussi bien par la guerre que par l'alliance matrimoniale. Les Dafālì s'inscrivent dans cette tradition en la diffusant et en la vivant à travers leur double fonction.

Celle-ci les met au service des dévots qui, selon le principe du système jajmānī, sont leurs patrons. Durant les deux fêtes, ils œuvrent pour eux comme « prêtre de pèlerinage » (pand $\bar{a})$, en les assistant aussi bien pour ce qui concerne l'« intendance » (intizām) que le « service rituel » $($ sevā $)$, afin qu' ils donnent en mariage Zohra Bībī à Ghāzī Miyāñ, en échange de ses faveurs, notamment de bonnes récoltes, dont ils lui offrent les prémices et les augures. Le service rituel des Dafālī dure du Cinquième jour de printemps à la Foire de mai-juin, c'est-à-dire durant la

24. Fuite de Mahomet et de ses partisans de la Mecque à Médine en 622. 
période où il est interdit aux proches du martyr (desservants, Dafālī et dévots) de se marier et, par conséquent, aux Dafālī de jouer de la musique pour les cérémonies de mariage du commun des mortels.

Pour leur service, les Dafālī reçoivent de leur patron un « présent » (nazrānah) qui consiste en une somme d'argent, et du sanctuaire les « charités » (sadaqe) de Ghāzī Miyāñn, comprenant le remboursement de leurs frais de déplacement, deux repas par jour le temps de leur séjour et 25 \% de la dot de Zohra Bībī, en échange de la diffusion de ses miracles et de la conduite des dévots et de leurs offrandes.

\section{Un culte qui ne va pas de soi}

Ce culte d'une figure de sainteté musulmane par une population largement hindoue ne va pas de soi et toutes les versions littéraires de sa légende visent à remettre en cause cet œcuménisme. La première, sur laquelle se fondent toutes les suivantes, est une hagiographie, le Miroir de Mas ${ }^{6} \bar{u} d$, composée en persan au XVII ${ }^{\mathrm{e}}$ siècle par un hagiographe professionnel, 'Abd al-Rahmān Ciśtīi ${ }^{25}$. Rédigée quatre siècles après le martyre, elle était destinée à l'élite musulmane de hautes castes, qui doutait alors de l'identité religieuse de Ghāzī Miyāñ en raison, justement, de son culte jugé trop hindou. Le but de l'hagiographe fut donc de légitimer le personnage, abscons aux yeux de cette élite, en donnant à son martyre une coloration que nous qualifierions aujourd'hui de jihadiste. Sous sa plume, Ghāzī Miyāñ prend les traits d'un combattant fanatique dont les exploits iconoclastes contre les hindous relèvent de la guerre sainte, à l'opposé donc des récits des desservants et des Dafālī.

À partir des années 1870, des vulgarisations en ourdou ${ }^{26}$ de ce texte sont régulièrement publiées, accompagnées d'embellissements poétiques. Puis dans les années 1910, des écrivains hindous produisirent à leur tour en hindi des adaptations brahmaniques de l'hagiographie, avec le souci de rectifier l'image de Ghāzī Miyāñ. Le mouvement revivaliste Ārya Samāj ${ }^{27}$ y participe dans une pers-

25. Comme son nom l'indique, il est membre de la confrérie Ciśtiyyā, dont le plus illustre représentant est $\mathrm{Mu}$ 'īnal-dīn Chishtī de Ajmer, d'où notre choix de faire référence à son sanctuaire dans nos références bibliographiques. Cet hagiographe va d'ailleurs œuvrer pour minimiser le rôle de Ghāzī Miyāñ dans la pénétration de l'islam dans le sous-continent indien au profit de son maître.

26. Langue accessible à un plus grand nombre que le persan.

27. L'Ārya Samāj ( «la Noble Société ») est un mouvement réformiste hindou créé en 1875 par Swami Dayananda Sarasvati (1824-1883). Elle prône le retour aux pratiques védiques et le prosélytisme de reconquête, c'est-à-dire la reconversion à l'hindouisme des musulmans et des chrétiens convertis ou issus de convertis. 
pective historique en faisant publier la Nouvelle histoire hindoue de Salār Mas ${ }^{6} \bar{u} d$. Cette version épouse le point de vue de l'hagiographie, qu'elle qualifie de texte historique d'origine et d'authentique version de l'histoire des musulmans en Inde, tandis qu'elle renvoie les ballades des Dafālī dans le domaine de la mystification, les dénonçant comme des histoires fabriquées par des vauriens de basse caste, dans le but de tromper les innocents hindous. Ces écrivains reprochent à la tradition orale de créer de la confusion entre les mondes islamique et hindou, alors qu'euxmêmes considèrent qu'hindous et musulmans ont élaboré en Inde deux cultures, deux sociétés, deux univers totalement différents. Ils promeuvent cette idée, à travers leur entreprise de dévoilement du vrai visage de Ghāzī Miyān que la tradition orale, selon eux, occulterait en dissimulant, par ruse, son identité, afin que les hindous ne sachent pas qu' ils ont affaire à des musulmans. Ils escomptent ainsi détourner les basses castes hindoues, et principalement les femmes, de l'influence des Dafālī et à terme leur faire abandonner ce culte. Tous ces livrets délivrent aux maris le même conseil : «Empêchez vos femmes de prier le Bābā, né d'une mère stérile, pour lui demander d'exaucer leur vœu d'une descendance mâle ». Le pamphlet rédigé par Nand Kishore Jaismal de Partapgarl en 1916, Ghazi Miyan or the real life story of Masaud Ghazi, en est un bon exemple : il raconte l'échec de Ghure Dafālī (littéralement Dafālī Tas de Fumier) devant ses patrons dans la joute oratoire qui l'oppose à un prêtre hindou. Plusieurs de ces versions sont aujourd'hui vendues, sous forme de petits livrets bon marché, sur les stands de la Foire de mai-juin.

À la même époque, les prêcheurs des mouvements réformistes et revivalistes musulmans (deobandī et barelvi ${ }^{28}$ ) ne furent pas en reste. Ils cherchèrent eux aussi à purifier la religion des basses castes mais cette fois des influences hindoues. La célébration de l'« anniversaire de naissance » (janām din) de Ghāzī Miyāñ fut alors instaurée par l'administration du sanctuaire (aux mains des hautes castes musulmanes depuis la première partie du $\mathrm{xx}^{\mathrm{e}}$ siècle), lors de laquelle des mollah, désavouant le culte aux saints (mais non les saints) ou cherchant à l'islamiser, sont invités à prêcher le 'vrai' islam et ses préceptes ; les desservants n'y participent pas

28. Le mouvement deobandī, apparu en 1867, s'inspire de la pensée de Shah Waliullah Dehlavi (1702-1762) influencée par le mouvement wahhabite saoudien. Le mouvement barelvī, fondé par Ahmed Raza Khan (1856-1921) en 1904, cherche lui à rendre plus orthopraxe l'islam traditionnel sud asiatique. Sur les mouvements réformistes indo-musulmans du $\mathrm{XIX}^{\mathrm{e}}$ siècle et du début du $\mathrm{XX}^{\mathrm{e}}$ siècle voir B. D. Metcalf (1982) et M. Gaborieau (2003 c). 
164 Des vies extraordinaires : les territoires du récit $-\mathrm{n}^{\circ} 79$

pour la raison, disent-ils, qu'ils n'y reconnaissent pas « l'ordre socio-cosmique du fort de l'ici du Bābā ».

Dans cet article, nous nous sommes attachée à montrer justement que cette religion du fort, qui repose sur la source bienfaitrice de la jungle de Bahraich, est expliquée et diffusée par les traditions orales, poétiques et naïves comme dirait R. Hertz, des desservants et des Dafālī. Leurs récits, pour donner un visage à cette source, ont créé une figure sainte dans laquelle les adeptes du culte peuvent reconnaître leur culture agricole et guerrière et leur société fondée sur la parenté et le système des castes. L'élaboration et la diffusion de ces narrations sont inséparables de la pratique de cette religion du fort, comme le montrent, d'une part, leurs acteurs et interprètes qui en sont aussi les officiants rituels et, d'autre part, les traditions savantes et édifiantes qui ne remettent pas en cause le personnage luimême mais la nature du culte qui lui est rendu. Justement parce que celui-ci n'est pas au service d'une religion instituée (islam ou hindouisme) mais d'une société régionale multiconfessionnelle qui cherche, à travers lui, la force de se perpétuer et de supporter les infortunes du sort.

\section{Bibliographie}

Ahmad, Imtiaz (éd), 1978 [1973], Caste and Social Stratification among Muslims in India, New Delhi: Manohar Book Service.

Ansari, Ghaus, 1960, Muslim Caste in Uttar Pradesh, Lucknow: Ethnographic and Folk Culture Society.

Amin, Shahid, 2005, « Un saint guerrier. Sur la conquête de l'Inde du Nord par les Turcs au XI ${ }^{\mathrm{e}}$ siècle $\gg$, Annales, nº 2, p. 265-292.

Bellamy, Carla, 2011, The Powerful Ephemeral. Everyday Healing in an Ambiguously Islamic Place, Berkeley: University of California Press, "South Asia across the disciplines".

Berque, Jacques, 2002, le Coran. Essai de traduction, Paris : Albin Michel («Spiritualités vivantes »).

Briggs, Geo. W, 1953, The Doms and their near Relations, Mysore: Wesley Press. 
Calavia Sáez, Oscar, 1996, Fantasmas falados: Mitos e mortos no campo religioso Brasileiro, Campinas : Editora da UNICAMP.

Centlivres, Pierre (éd.), 2001, Saints, Sainteté et Martyre. La fabrication de l'exemplarité, Neuchâtel : Éditions de l'Institut d'ethnologie/Paris : Éditions de la Maison des sciences de l'homme, « Recherches et travaux de l'Institut d'ethnologie $\gg$.

Chambert-Loir, Henri et Guillot, Claude (dir.), 1995, le Culte des saints dans le monde musulman, Paris : Éditions de l'École française d'Extrême-Orient (Études thématiques 4).

Crooke, William, 1968 [1896], The Popular Religion and Folklore of Northern India (2 vol.), Delhi: Munshiram Manoharlal.

Currie, P. Mark, 1989, The shrine and Cult of Muin al-din Chishti of Ajmer, Delhi: Oxford University Press.

Falasch, Ute, 2016, "Negotiating Religious Authority at a Shrine Inhabited by a Living Saint - The dargāh of "Zinda" Shāh Madār", in M. Boivin and R. Delage (dir.), Devotional Islam in Contemporary South Asia: Shrines, Journeys and Wanderers, New Delhi: Routledge, pp. 63-77.

Fox, James J., 2002, "Interpreting the historical significance of tombs and chronicles in contemporary Java”, in H. ChamberT-Loir et A. Reid (dir.), The Potent Dead. Ancestors, Saints and Heroes in contemporary Indonesia, Crows Nest, NSW: Asian Studies Association of Australia in association with Allen/ Unwin, Honolulu: University of Hawai'i, pp. 161-172.

Gaborieau, Marc, 1993a, Ni brahmanes ni ancêtres. Colporteurs musulmans du Népal. Nanterre, Société d'Ethnologie.

Gaborieau, Marc, 2003b, "Introduction To The New Edition", in Christian Troll (dir.), Muslim Shrines in India: Their Character, History and Significance, New Delhi, Oxford University Press, pp. V-XXi.

Gaborieau, Marc, 2003c, "A Nineteenth-Century Indian "Wahhabi" Tract Against the Cult of Muslim Saints: Al-Balagh al-Mubin”, in C. Troll (dir.), Muslim Shrines in India: Their Character, History and Significance, New Delhi, Oxford University Press, pp. 198-239. 
Greeven, Richard, 2010 [1898], The Heroes Five (Panchon Pir). An Attempt to Collect Some of the Songs of the Pachpirya Ballad-Mongers in the Benares Division, Memphis: General Books.

Herklots, Gerhard Andreas (composed under the direction of and translated by), 1972 [1921], Islam in India or the Qānün-i-Isläm by Ja'far Sharif, Oxford: Oxford University Press.

Hertz, Robert, 1913, « Saint Besse, étude d'un culte alpestre », Revue de l'histoire des religions, LXVII, p. 115-180.

Jamous, Raymond, 1995, « "Faire", "défaire" et "refaire" les saints : Les pir chez les Meo (Inde du Nord) », Terrain, vol. 24, p. 43-56.

Lindholm, Charles, 1986, "Caste in Islam and The problem of deviant systems: a critique of recent theory", Contributions to Indian sociology, vol. 20, ${ }^{\circ} 1$, pp. 61-73.

Mann, Elizabeth A., 2003 [1989], "Religion, Money and Status: Competition for Resources at the Shrine of Shah Jamal, Aligarhculte”, in Christian Troll (dir.), Muslim Shrines in India: Their Character, History and Significance, New Delhi, Oxford University Press, pp. 145-171.

Metcalf, Barbara D., 1982, Islamic Revival in British India: Deoband, 18601900, Princeton: Princeton University Press.

Morni, Syed Liyaqat Hussain, 2003, "Rituals and Customary Practices at the Dargah of Ajmer", in Christian Troll (dir.), Muslim Shrines in India: Their Character, History and Significance, New Delhi, Oxford University Press, pp. 60-75.

Ortis, Delphine, 2016a, "How Discourses and Rituals Construct Figures of Holiness: The Example of the Indo-Muslim Martyr Ghāzī Miyān (Uttar Pradesh, North India)", in M. Boivin et R. Delage (dir.), Devotional Islam in Contemporary South Asia: Shrines, Journeys and Wanderers, New Delhi: Routledge, pp. 79-99. 
Ortis, Delphine, 2016b « Comment des hindous peuvent-ils être les dévots d'un guerrier musulman ? Pour une relecture de la geste de Ghāzī Miyāñ à la lumière de ses attributs et de son culte », Journal asiatique 304.1 (2016), p. 73-91.

Ortis, Delphine, 2016c (à paraître), "From Potent Dead to Potent Places? Reflections on the Shrines of the Muslim Saints in South Asia", in Asia and Pacific Journal of Anthropology, Canberra: Routledge.

Pinch, William R., 1996, Peasants and monks in British India, Berkeley/Los Angeles/London: University of California Press.

Servan-Schreiber, Catherine, 1999, Chanteurs itinérants en Inde du Nord. La tradition orale Bhojpuri, Paris: L'Harmattan, « Recherches asiatiques ».

SIKAND, Yoginder, 2004, "Shared Hindu-Muslim Shrines in Karnataka: Challenges to Liminality”, in Ahmad Imtiaz et Reifeld Helmut (dir.), Lived Islam in South Asia. Adaptation, Accommodation and Conflict, Delhi: Social Science Press, pp. 166-186.

Speziale, Fabrizio, 2010, Soufisme, religion et médecine en Islam indien, Paris : Éditions Karthala.

Temple, Richard Carnac, 1884, The Legends of the Panjâb, Bombay: Education Society's Press.

VON SCHWERIN, Kerrin Gräfin, 1981, "Saint Worship in Indian Islam: The Legend of the Martyr Salar Masud Ghazi”, in I. Ahmad (dir.), Ritual and Religion among Muslims in India, New Delhi: Manohar, pp. 143-161.

Résumé : dans cet article portant sur l'une des figures de sainteté musulmane les plus anciennes et populaires de l'Inde du Nord, Ghāzī Miyāñ, sont étudiées les fonctions remplies par les traditions orales diffusées, par les desservants de son tombeau et les musiciens dafälī, lors des rites quotidiens et festifs. Cette double tradition est mise en regard des récits littéraires allant à son encontre pour s'opposer à ce culte. Notre visée est de montrer que l'ensemble de ces récits, bien qu'ils semblent nous conter les hauts faits de sa vie justifiant son statut de martyr, s'élaborent en fait en s'adossant au culte, dans le but de le légitimer ou de le détruire.

Mots clefs : légende, rituel, Inde, Uttar Pradesh, anthropologie, culte des saints, hindouisme, islam 
168 Des vies extraordinaires : les territoires du récit $-\mathrm{n}^{\circ} 79$

\section{The Role of Legend in the Cult of Ghäzì Miyān}

Abstract: This article focuses on one of northern India's earliest and most popular Muslim saintly figures, Ghäzi Miyān, and the role oral tradition plays in the daily and festive rituals performed in his honour by the custodians of his tomb and dafāli musicians. This dual tradition contrasts with literary tales unfavourable to him and that are used to denigrate the cult. Our intention is to show that all these stories, although they seem to describe key events of his life justifying his status as a martyr, are in fact rooted in the cult with the aim of either legitimizing or of destroying it.

Keywords: legend, ritual, India, Uttar Pradesh, anthropology, cult of the Saints, Hinduism, Islam

\section{Note sur l'auteur}

Après l'obtention d'une thèse en anthropologie sociale et ethnologie à l'EHESS portant sur le culte du martyr Ghāzī Miyāñ, Delphine Ortis poursuit ses recherches au Pakistan sur la fabrication de la sainteté indo-musulmane, l'organisation des sanctuaires et les liens entre soufisme et chiisme. Elle est actuellement chargée de cours en anthropologie à l'ESSEC, l'Inalco et l'EHESS. 